\title{
Electrophysiology and Dye-Coupling Are Sexually Dimorphic Characteristics of Individual Laryngeal Muscle Fibers in Xenopus laevis
}

\author{
Martha L. Tobias and Darcy B. Kelley \\ Department of Biological Sciences, Columbia University, New York, New York 10027
}

Sex differences at the laryngeal neuromuscular junction of Xenopus laevis were examined by recording intracellularly from muscle fibers in response to nerve stimulation. Male laryngeal muscle contains 2 physiologically distinct fiber types. Type I fibers generate postsynaptic potentials in response to low-magnitude stimulus pulses and action potentials in response to higher-magnitude stimulus pulses. Type II muscle fibers require repetitive stimulation for action potential production, probably because of facilitation. Subthreshold events in type I and II fibers suggest that these neuromuscular synapses have low safety factor junctions. Female laryngeal muscle contains one fiber type (III), which is physiologically distinct from those found in the male. Type III fibers produce an action potential in response to a singlestimulus pulse of suprathreshold voltage delivered to the laryngeal nerve; subthreshold events were not observed. lontophoretic injection of Lucifer yellow into a single female muscle fiber resulted in as many as $\mathbf{4 3}$ labeled fibers. In males, only one fiber was labeled. Dye-coupling was not observed in adult females treated with the androgenic steroid hormone, testosterone. We have previously reported that laryngeal muscle fibers are recruited throughout a stimulus train presented to the laryngeal nerve in males, but are not recruited in females (Tobias and Kelley, 1987). Sex differences in the frequency of electrophysiological fiber types described here may account for sex differences in fiber recruitment. Synchronous activity of dye-coupled fibers may increase the effectiveness of muscle contraction in females.

The clawed frog, Xenopus laevis, relies on vocal behavior to advertise reproductive state. Vocalizations are sexually dimorphic in rate, temporal pattern, and function (Wetzel and Kelley, 1983). The male-specific mate call-a rapid trill with alternating fast and slow click rates - attracts and excites females (Picker, 1983). The female-typical call, ticking-a slow, monulonous trill-is used to terminate male clasp attempts (Weintraub et al., 1985). Sounds are produced when laryngeal muscles contract, pulling open 2 apposed discs of arytenoid cartilage (Yaeger, 1982). These distinctive vocalizations differ in requirements for laryngeal muscle activity. Mate calling requires laryngeal muscles to contract rapidly $(71 \mathrm{~Hz}$ ) for long periods (Wetzel and Kelley, 1983). Ticking requires laryngeal muscles to contract

\footnotetext{
Received July 8, 1987; revised Dec. 4, 1987; accepted Dec. 10, 1987

This work was supported by NIH Grants NS 23684 and NS 07685 .

Correspondence should be addressed to Dr. Tobias, Department of Biological Sciences, 909 Fairchild, Columbia University, New York, NY 10027.

Copyright (C) 1988 Society for Neuroscience $0270-6474 / 88 / 072422-08 \$ 02.00 / 0$
}

more slowly $(6 \mathrm{~Hz})$ for short periods (Hannigan and Kelley, 1986).

Sex differences in behavior can be due to male/female dimorphisms in the CNS and/or the periphery. Laryngeal motor neurons (Simpson et al., 1986; Kelley et al., 1988), CNS pathways (Wetzel et al., 1985), and laryngeal muscle (Sassoon and Kelley, 1986; Sassoon et al., 1987; Segil et al., 1987) are all sexually dimorphic candidates for producing sex differences in vocal behavior. Recent studies in the isolated larynx, examining muscle activity in response to nerve stimulation (Tobias and Kelley, 1987), reveal that at least 2 properties of call production are regulated in the periphery: the rate of muscle contraction, which controls the rate of click production, and the strength of muscle contraction, which contributes to click amplitude. The rate at which discrete tension transients (muscle contractions that increase and decrease to resting level) are produced by laryngeal muscle in response to nerve stimulation is considerably faster in males than in females. Histochemical characteristics of laryngeal muscle are consistent with the dimorphism in contraction rate. Male laryngeal muscle is composed entirely of fast-twitch, highly oxidative fibers, while female laryngeal muscle is composed primarily of slow, moderately oxidative fibers (Sassoon et al., 1987). The size of muscle tension transients is controlled by the number of muscle fibers contracting simultancously, a property reflected in the amplitude of muscle compound action potentials. In male laryngeal muscle, nerveevoked compound action potential amplitudes increase dramatically throughout the entire stimulus train. In female laryngeal muscle, compound action potential amplitudes increase significantly less and only at the beginning of the stimulus train. The magnitude of potentiation is directly proportional to stimulus frequency in males and inversely proportional in females. Since each compound action potential is the summed response of all muscle fibers responding to a given stimulus pulse, we suggest that only in males are muscle fibers recruited throughout the stimulus train. Possible sites for regulation of muscle fiber recruitment in the isolated larynx are the muscle fiber membrane or laryngeal motor neuron terminals.

One goal of the present study was to examine possible sex differences in the electrophysiological properties of laryngeal muscle fibers. These experiments extend our previous work (Tobias and Kelley, 1987) to the cellular level. In addition, we wished to examine mechanisms underlying sex differences in fiber recruitment. We report here that laryngeal muscle is composed of 3 electrophysiologically distinct fiber types, which can be classified as male- or female-like on the basis of their relative abundance in the 2 sexes. The frequency and characteristics of 
each fiber type within a sex are consistent with observed sex differences in fiber recruitment. Androgen treatment of adult females does not significantly increase the proportion of malelike fibers. The relative contributions of pre- and postsynaptic mechanisms to observed electrophysiological fiber types are discussed.

A second goal of this study was to examine the relation between muscle fiber types as characterized electrophysiologically (this study) and histochemically (Sassoon et al., 1987). Studies of ATPase and SDHase expression in laryngeal muscle show that fiber diameter is correlated with twitch type (Sassoon et al., 1987). Thus, to compare electrophysiological fiber type with diameter, we injected individual fibers with the low-molecularweight dye Lucifer yellow (Stewart, 1978). We have found that, in females, filling a single fiber with dye can result in the labeling of many tibers of different diameters. In males, dye filling a single fiber always results in only one labeled fiber. The presence of dye-coupling in laryngeal muscle appears to be androgenregulated, since coupled fibers have not been observed in male or in testosterone-treated female laryngeal muscle. We hypothesize that adult female laryngeal muscle fibers form functional syncytia that can be disrupted by the androgenic steroid hormone testosterone.

\section{Materials and Methods}

Adult Xenopus laevis were obtained from Xenopus I (Ann Arbor, MI) or Nasco (Fort Atkinson, WI). Animals were maintained on a 12:12 hr light: dark cycle in plastic tanks, and fed frog brittle every other day. Some adult females were briefly anesthetized with cold and received a $16-20 \mathrm{mg}$ implant of testosterone propionate (Sigma) into the dorsal lymph sac for 1 month. Females in this study were not ovariectomized because a previous study had shown no differences in laryngeal muscle physiology (compound action potential potentiation and contraction rates) between intact, hormone-implanted and ovariectomized, hormone-implanted females (Tobias and Kelley, 1987). Prior to dissection, all frogs werc ancsthetizcd by immersion in ethyl $m$-aminobenzoate methanesulfonic acid $(0.1 \%$; Aldrich). The larynx was excised as previously described (Tobias and Kelley, 1987), pinned dorsal side up in a wax-coated petri dish, and bathed in glucose-supplemented amphibian saline solution $(\mathrm{NaCl}, 0.1 \mathrm{M} ; \mathrm{KCl}, 2 \mathrm{~mm} ; \mathrm{CaCl}, 2.5 \mathrm{~mm} ; \mathrm{MgCl}, 3.0 \mathrm{~mm}$; HEPES, $4 \mathrm{~mm}$; glucose, $27.7 \mathrm{~mm}$ ). Laryngeal muscle is capable of strong contractions for several hours under these conditions (Tobias and Kelley, 1987). Individual muscle fibers were impaled with glass microelectrodes filled with either potassium acetate $(2.5 \mathrm{M})$ or Lucifer yellow $(4 \%$ in $0.1 \mathrm{M} \mathrm{LiCl}$ ). Electrode resistance ranged from 25 to $45 \mathrm{M}$. Recordings were amplified (Getting, model 5A) and photographed directly from a storage oscilloscope (Tektronix 5113). Only fibers with resting potentials greater than $80 \mathrm{mV}$ were examined. The laryngeal nerve was stimulated via a suction electrode (WPI 302-T). The stimulus voltage was gradually increased until a muscle fiber response was clicited. Stimulus trains werc $200 \mathrm{msec}$ in duration and varied in frequency from 25 to $66 \mathrm{~Hz}$. All stimulus trials were separated by $10 \mathrm{sec}$, to avoid facilitation or summation at the postsynaptic membrane.

Lucifer yellow was iontophoretically injected $(5-10 \mathrm{nA}, 500 \mathrm{msec} /$ $\mathrm{sec}$ ) into single fibers. Larynges containing dye-filled cells were placed in fresh $4 \%$ paraformaldehyde $(0.1 \mathrm{~m}$ phosphate buffer) for $3 \mathrm{hr}$, followed by $20 \%$ sucrose/paraformaldehyde overnight. Tissue was cryostat-sectioned $(25 \mu \mathrm{m})$, dehydrated, cleared in xylene, and mounted in a nonfluorescent medium (Lustrex; Monsanto). Sections were examined with a Zeiss fluorescence microscope. The significance of the differences in frequency of fiber types encountered was evaluated using the chi-square test (Siegel, 1956). The sex differences in the frequency of dye-coupled fibers were evaluated using the Fisher exact probability test (Siegel, 1956).

\section{Results}

Physiological sex differences in laryngeal muscle fibers

Males. Male laryngeal muscle contains 2 electrophysiologically identifiable fiber types. Type I fibers generate action potentials
A<smiles>C=C=C=CC</smiles>
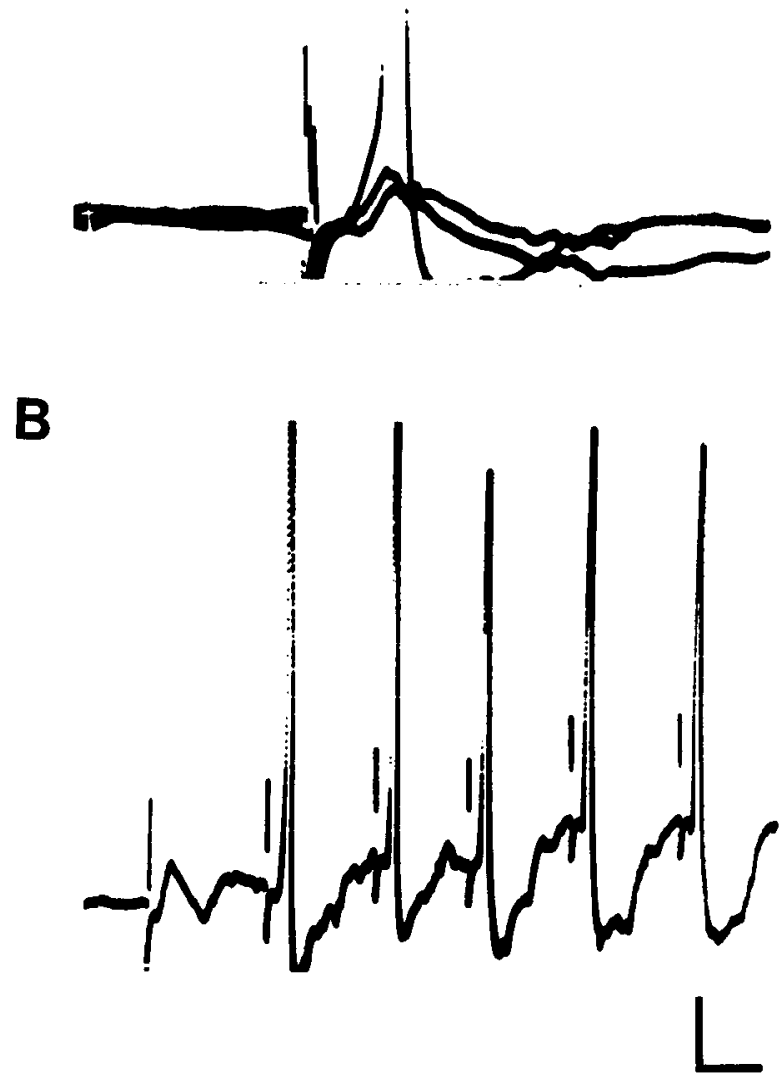

Figure 1. Response of a type I fiber to stimulation of the laryngeal nerve in male larynx. $A$, Response of the type I fiber to single-stimulus pulses of different strengths. 'Two subthreshold events of similar amplitude are produced in response to 2 different low-voltage stimuli. A third, higher-voltage stimulus results in production of an action potential. The top of the action potential is off-screen at this gain. Calibration bars: $10 \mathrm{mV}$ (vertical), $5 \mathrm{msec}$ (horizontal). $B$, Response of a type I fiber to stimulus trains of subthreshold voltage delivered to the laryngeal nerve. This fiber produces action potentials in response to each stimulus pulse within the train after an initial subthreshold event. Calibration bars: $10 \mathrm{mV}$ (vertical), $20 \mathrm{msec}$ (horizontal).

in response to single-stimulus pulses delivered to the laryngeal nerve, while type II fibers generate action potentials only in response to stimulus trains. Responses of a type I fiber are illustrated in Figure 1. A low-voltage stimulus pulse presented to the laryngeal nerve results in a single subthreshold event in the muscle fiber (Fig. 1 $A$ ). As the voltage is increased, a postsynaptic potential (PSP) of similar amplitude is produced. Still higher-voltage stimulation of the laryngeal nerve results in production of an action potential. The amplitude of the response, PSP, or action potential, is solcly dependent on stimulus strength, regardless of the order in which the stimulus pulses are presented. Stimulus trains at voltages subthreshold for action potential generation initially produce a PSP, followed by action potentials (Fig. 1B). Action potentials are then generated in response to each stimulus pulse within the train. 
A
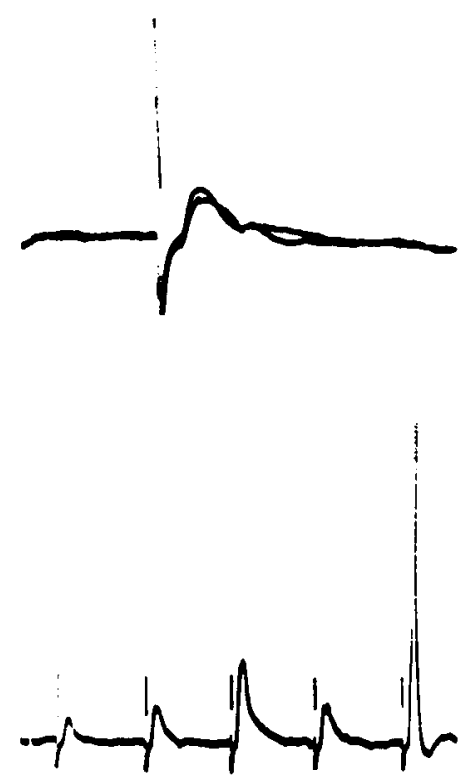

$43 \mathrm{~ms}$

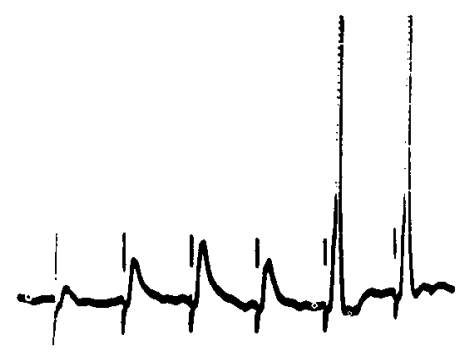

$36 \mathrm{~ms}$

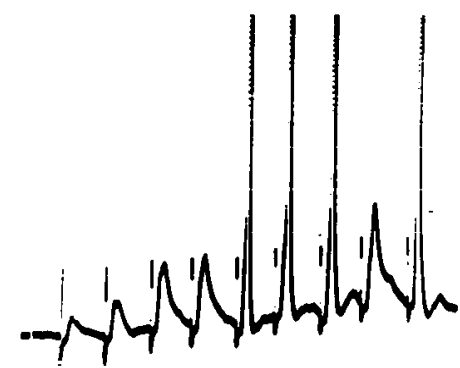

$20 \mathrm{~ms}$
B
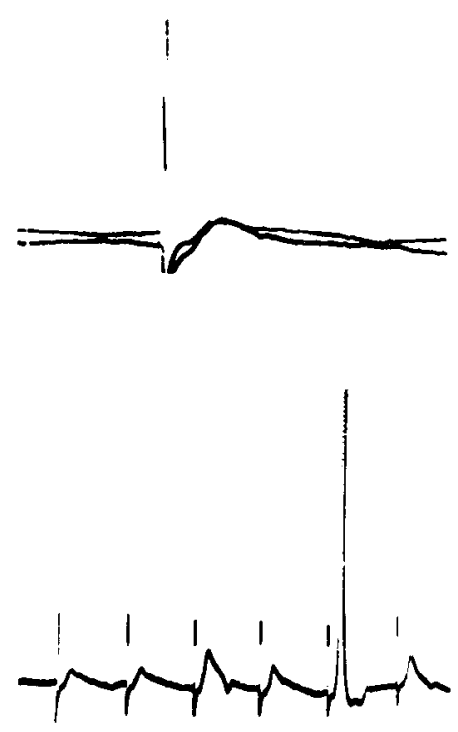

$36 \mathrm{~ms}$

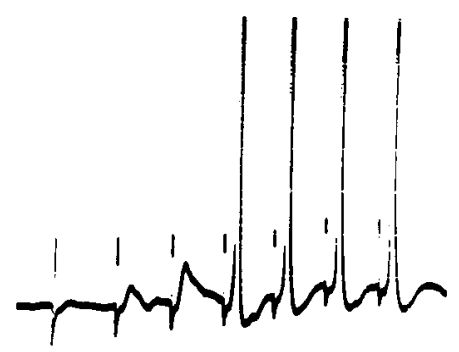

$30 \mathrm{~ms}$

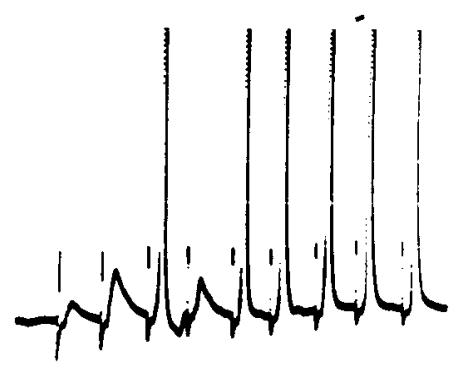

$20 \mathrm{~ms}$ msec (lower 3 panels). $m s$, mil onds.

Type II fibers produce only subthreshold events in response to single-stimulus pulses delivered to the laryngeal nerve (Fig. 2). The amplitude of the PSP is the same regardless of stimulus strength. Stimulus pulses were presented at the same magnitude as, or greater than, those required to elicit an action potential in type I fibers. Type II fibers vary in the extent to which they are frequency-dependent; 2 examples are shown in Figure 2. In the frequency-sensitive fiber, action potentials are produced after fewer subthrehsold events as the interpulse interval is decreased (Fig. $2 B$ ). In the frequency-insensitive fiber, action potentials are produced after the same number of subthreshold events over a similar range of stimulus frequencies (Fig. 2A).
PSP amplitude tends to increase with succeeding stimuli within a train, indicating facilitation. Type II fibers do not generate an action potential in response to every stimulus pulse within a train, even after the initial action potential is produced.

Females. Female laryngcal muscle contains predominantly one fiber type (type III), which is electrophysiologically distinguishable from those types found in male laryngeal muscle. Type III fibers produce an action potential in response to single-stimulus pulses of suprathreshold voltage delivered to the laryngeal nerve (Fig. $3 A$ ). Subthreshold events were not observed in the 38 fibers examined. Stimulus trains elicit an action potential in response to each pulse within the train (Fig. $3 B$ ). We have ob- 
Table 1. Incidence of physiological fiber type and dye-coupling in male, female, and testosterone-treated female frogs

\begin{tabular}{|c|c|c|c|}
\hline Type & $\begin{array}{l}\text { Male } \\
(\%)\end{array}$ & $\begin{array}{l}\text { T-Female } \\
(\%)\end{array}$ & $\begin{array}{l}\text { Female } \\
(\%)\end{array}$ \\
\hline I & $11(25.0)$ & $2(12.5)$ & $2(4)$ \\
\hline II & $31(70.5)$ & $2(12.5)$ & $7(15)$ \\
\hline III & $2(4.5)$ & $12(75.0)$ & $38(81)$ \\
\hline Total ${ }^{a}$ & 44 & 16 & 47 \\
\hline Dye-coupled/[total $\left.{ }^{b}\right]$ & $0[15]$ & $0[8]$ & $8^{c}[20]$ \\
\hline
\end{tabular}

a Total number of fibers examined in 20 males, 8 testosterone-treated females, and 21 females.

${ }^{b}$ Instances of dye-coupled fibers [total number of fibers injected]. This experiment was performed in 9 males, 5 testosterone-treated females, and 9 females.

- The frequency of dye-coupling in females is significantly greater than that in males (Fisher exact probability test; $p=0.017$ ). In 2 of the 8 cases in which coupling was observed, only 2 fibers were coupled. These cases were not included in the statistical analysis.

served 1:1 following at stimulus frequencics as fast as $66 \mathrm{~Hz}$ (data not shown). Both type I (male-typical) and type III (femaletypical) fibers are similar in that they produce an action potential in response to a single-stimulus pulse. In addition, both fiber types follow pulses 1:1 within a stimulus train. However, only type I fibers produce subthreshold events in response to lowvoltage stimulus pulses.

The designation of a fiber type as male-iike is based on the fact that such fibers (types I and II) appear more frequently in males than in females $(p<0.001$ and $p<0.01$, respectively; Table 1). Similarly, the female-like type III muscle fiber is found more frequently in females than in males $(p<0.001)$. Although each fiber type is characteristic of only one sex, all fiber types are present in small numbers in both sexes. However, the probability that a type III or type I fiber will be found in the atypical sex is significantly less than the probability that the male-typical type II fiber will be found in a female $(p<0.001)$. Thus, types $I$ and III fibers are the most, and type II fibers the least, sexually dimorphic. In summary, physiological fiber type is sex-typical, not sex-specific.

\section{Sex differences in dye-coupling of laryngeal muscle fibers}

In some preparations, a Lucifer yellow-filled electrode was used to iontophoretically inject dye into a muscle fiber following electrophysiological characterization. Dye injection into male laryngeal muscle fibers resulted in only one labeled cell in all fibers examined (Fig. 4A, Table 1). In contrast, dye injection into a female laryngeal muscle fiber sometimes (in 8 out of 20 cases) resulted in multiple labeling (Fig. $4 B$, Table 1). Both type I and type III fibers were dye-coupled. Both small and large fibers were dye-coupled. Thus, it is likely that fibers of various physiological types are coupled together. The number of labeled cells in the dye-coupled preparations varied from 2 to 43 . Laryngeal muscle fibers are organized in an anterior-to-posterior direction. Dye-coupled fibers are organized in rows; any one fiber is offset dorsoventrally from its medial neighbor (Fig. 4B).

The original purpose of this experiment was to determine whether fiber diameter is correlated with electrophysiological fiber type in female larynx. In those cases where dye-filling an individual fiber resulted in multiple-cell labeling, the diameter of the impaled cell could not be determined. Fiber diameters of 3 single-labeled fibers were compared to those of other fibers in the same muscle. In 2 preparations, the dye-filled fiber was
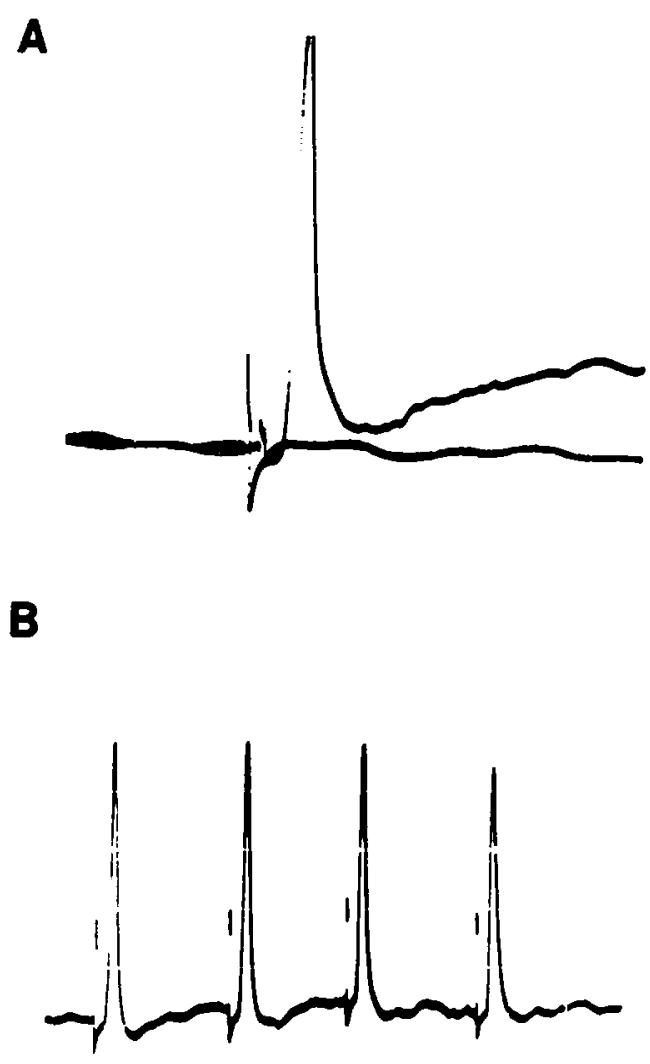

Figure 3. Response of a type III fiber to nerve stimulation in female larynx. $A$, Response of a type III fiber to single-stimulus pulses. No response is observed in response to a subthreshold stimulus. Suprathreshold stimuli result in an action potential. Calibration bars: $10 \mathrm{mV}$ (vertical), 5 msec (horizontal). $B$, Response of a type III fiber to stimulus trains. An action potential is produced in response to each stimulus pulse within a train. The stimulus voltage was the same as that eliciting an action potential in $A$. Calibration bars: $10 \mathrm{mV}$ (vertical), $20 \mathrm{msec}$ (horizontal).

similar in diameter to the largest fibers in the same muscle. In one preparation, the dye-filled fiber was similar in diameter to medium-sized fibers in the same muscle. Thus, large- and medium-sized fibers were impaled with Lucifer yellow-filled electrodes.

\section{Effects of testosterone on female laryngeal muscle fibers}

Testosterone treatment of adult females results in partial masculinization of calling behavior (Hannigan and Kelley, 1986), of muscle fiber size and histochemical fiber types (Sassoon et al., 1987), and of laryngeal muscle tension characteristics and compound action potential amplitude (Tobias and Kelley, 1987). An increase in the percentage of male-typical physiological fiber types could help to explain the masculinization of compound action potential amplitude potentiation (Tobias and Kelley, 1987). The frequency of male-typical muscle fibers (types I and II) is not significantly different in control and testosterone-treated females. Testosterone-treated females continue to exhibit a predominance of type III fibers.

Lucifer yellow injection into laryngeal muscle fibers from testosterone-treated females resulted in only one labeled cell per 


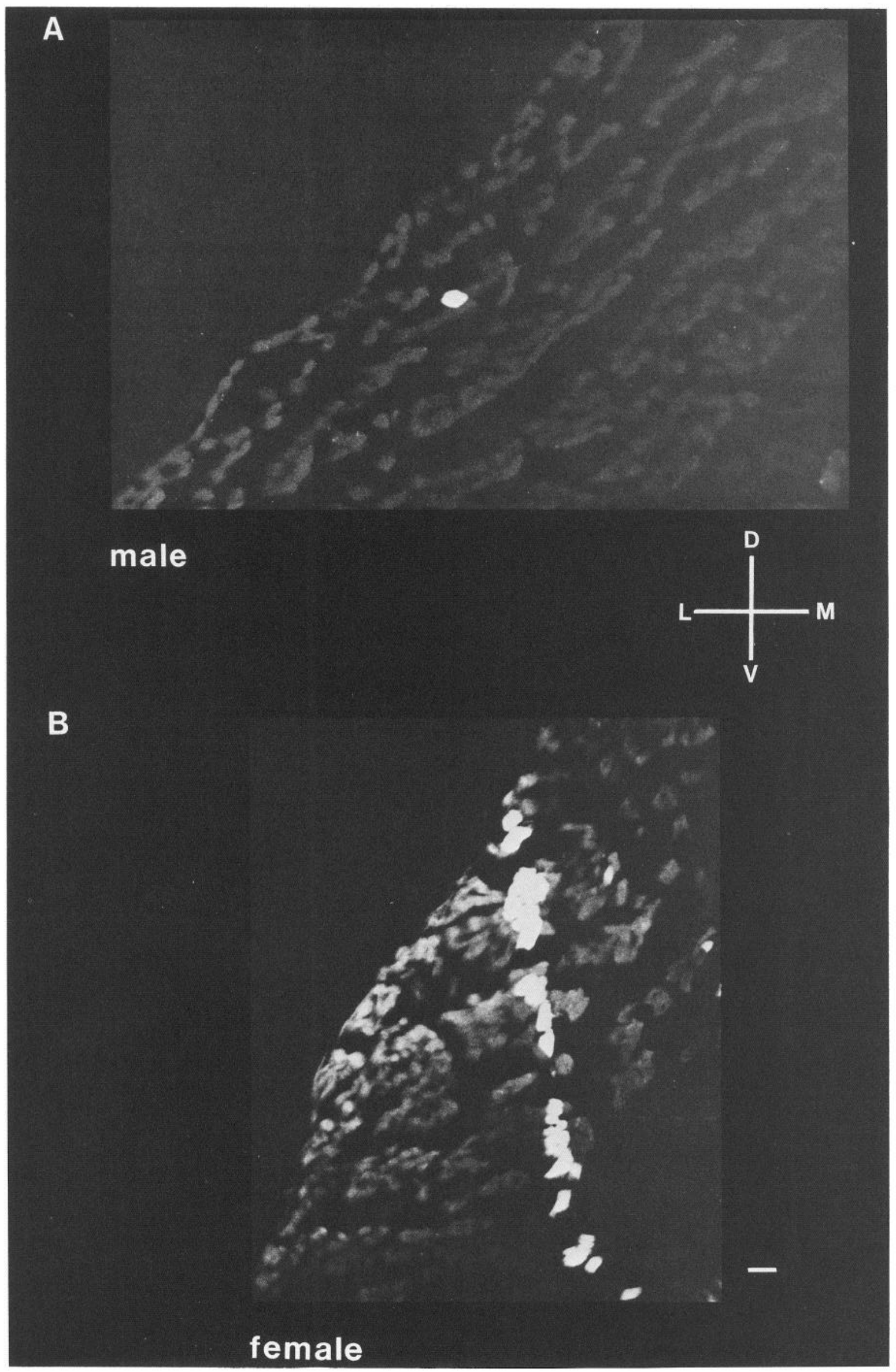


preparation in each of 8 preparations (Table 1). Since dye-coupling is observed in 8 out of 20 untreated females, testosterone appears to reduce coupling between muscle fibers in females.

\section{Discussion}

Male and female fiber types and laryngeal function

Male and female Xenopus laevis laryngeal muscle fibers respond to nerve stimulation in a sex-typical manner. Three physiologically distinct fiber types have been described. Fiber types I and II are found predominantly in males, and type III is found predominantly in females. Both male-typical fiber types generate subthreshold potentials in response to nerve stimulation. The female-typical fiber type responds to nerve stimulation in an all-or-none fashion; subthreshold potentials are never observed.

\section{Fiber recruitment in males and females}

One goal of this study was to account, at the cellular level, for sex differences in muscle fiber recruitment (Tobias and Kelley, 1987). In males, laryngeal muscle fibers are recruited throughout a stimulus train, while in females, muscle fibers are recruited only at the beginning of a stimulus train. The result is dramatic potentiation of compound action potentials in males but not in females. The predominance of type III fibers in females might contribute to the constancy of compound action potential amplitude. Type III fibers respond in an all-or-none fashion to each pulse presented to the laryngeal nerve. Once threshold for generation of an action potential is attained, spikes are faithfully produced throughout a stimulus train. The coupling observed between female fibers might contribute to this result, since all coupled fibers would produce an action potential nearly synchronously. Male laryngeal muscle contains predominantly 2 fiber types, I and II. Type I fibers, like type III fibers, produce action potentials in response to each suprathreshold pulse within a stimulus train. Thus type I fibers contribute to the baseline amplitude of the compound action potential in males. In contrast to females, however, males also possess a large proportion of type II fibers, which are recruited as the stimulus train proceeds. Since type II fibers produce action potentials only in response to repetitive nerve stimulation, they add to the response generated by type I fibers, effectively increasing the compound action potential amplitude as the train proceeds. Each type II fiber produccs an action potential after a different number of subthreshold events (see Fig. 2 for 2 examples). The frequency dependence of many type II fibers may also explain why compound action potential amplitude increases with stimulus frequency (Tobias and Kelley, 1987).

\section{Pre-versus postsynaptic contribution to muscle fiber type}

Both male laryngeal muscle fiber types (I and II) produce subthreshold potentials in response to nerve stimulation. Analysis of miniature endplate potentials in frog sartorius and $\mathrm{Cu}$ taneous pectoris muscles reveals that individual synapses frequently produce only PSPs, not action potentials. Fewer quanta of neurotransmitter are released at low than at high safety factor junctions, consistent with differences in calcium availability within the nerve terminal (Grinnell and Herrera, 1980). Thus, the presence of subthreshold muscle responses can reflect a presynaptic property: release of small amounts of transmitter. In Xenopus laevis larynx, type II muscle fibers require repetitive stimulation by the laryngeal nerve for the production of action potentials. The increased size of PSPs during a stimulus train is probably due to facilitation, rather than to temporal summation, at the muscle fiber membrane. The latter mechanism could operate only if the interstimulus interval were less than the duration of a PSP, resulting in summation. In our experiments, the membrane potential always returned to resting level prior to the next response. Thus, we suggest that type II muscle fibers produce action potentials in response to increased transmitter release at the presynaptic terminal during repetitive nerve stimulation. Only one electrophysiologically identifiable fiber type has been described in female laryngeal muscle (type III). Type III fibers are typical of most vertebrate skeletal muscle: enough transmitter is released in response to each motor neuron action potential to guarantee a postsynaptic action potential. Apparent differences in transmitter release at the presynaptic terminal in males and females suggest that laryngeal motor neurons may also be physiologically different in the 2 sexes.

We have considered whether the detection of subthreshold events in male-typical fiber types only might result from sex differences in the location of neuromuscular junctions within the muscle, or from sex differences in muscle membrane properties. Karnovsky staining of laryngeal muscle has revealed that endplates are located in identical positions for males and females, the middle of the laryngeal muscle at all anterior/posterior levels (unpublished observations). This staining pattern is identical in both sexes. Since the recording electrode was randomly placed in the muscle in all experiments, electrode position probably does not account for sex differences in fiber type observed in this study. However, even if the electrode were the same distance from the endplate in both sexes, subthreshold events might be recorded only in males if the space constant of the membrane in males were larger. Fiber diameter and space constant are proportional; passive electrical events decay over a shorter distance in smaller fibers. Given the small diameter of most male laryngeal muscle fibers (Sassoon et al., 1987), it is unlikely that the smallest laryngeal muscle fibers are routinely, let alone exclusively, encountered in the female (see below).

\section{Lack of correlation between histochemical and electrophysiological fiber types}

Male laryngeal muscle is comprised of a homogeneous population of fast-twitch, highly oxidative (fatigue-resistant) fibers (Sassoon et al., 1987). In contrast, female laryngeal muscle is comprised of 3 histochemically identifible fiber types: small, slow-twitch, highly oxidative (74\%); medium-sized, fast-twitch, highly oxidative (6\%); and large, fast-twitch, moderately oxidative (20\%; Sassoon et al., 1987). We have described at least 2 electrophysiologically distinct fiber types in male larynx and one in female larynx. Thus, male larynx is homogeneous his-

Figure 4. Photomicrographs of Lucifer yellow-labeled fibers in laryngeal muscle. The entire larynx was transversely sectioned ( $25 \mu \mathrm{m})$. Labeled fibers could be reliably followed from section to section for distances up to $1 \mathrm{~mm}$. Calibration bar, $30 \mu \mathrm{m}$. $A$, A single fiber is labeled following dye injection into an individual fiber in a male larynx. $B$, Multiple labeling observed following dye injection into an individual fiber in a female larynx. The fiber injected was within the superficial (i.e., lateral) third of the muscle leaflet shown in the photomicrograph. Muscle fibers of different diameters are dye-coupled. 
tochemically and heterogeneous electrophysiologically, while female larynx is heterogeneous histochemically and homogeneous electrophysiologically.

It is possible that predominantly large fibers, all of which may have the same physiological characteristics, are encountered in the intracellular experiments on females. Two size classes, medium and large, are included in the single dye-labeled fibers we examined. Thus, we may have sampled 2 histochemical fiber types that constitute only one electrophysiological type. The issue of whether male-typical fibers are abundant but undetected in females remains unresolved. However, the fact remains that female-typical fibers are extremely rare in males.

\section{Multiple innervation of laryngeal fibers; androgen and synapse elimination}

Male-typical type I fibers exhibit a subthreshold event in response to a low-voltage stimulus delivered to the laryngeal nerve and an action potential in response to a higher-voltage stimulus, suggesting that these fibers are innervated by at least 2 motor neurons. The absence of subthreshold potentials in type III fibers does not prove that these fibers are singly innervated. In Xenopus laevis pectoral muscle, terminals with a low safety factor are typically associated with multiply innervated endplates. The sum of quanta released is equivalent to that of a singly innervated endplate (Angaul-Petit and Mallart, 1979). The persistence of multiple innervation in adults is due to the failure to eliminate nerve terminals during the normal course of muscle development (Bennett and Pettigrew, 1975; Brown et al., 1976). Testosterone-regulated synapse elimination has been postulated to operate in the levator ani, an androgen-sensitive muscle of rodents (Jordan et al., 1988). In this muscle, multiple innervation of single fibers, as well as of single endplates, is found in developing males at times when other skeletal muscles have eliminated supernumerary synapses. The muscle is lost postnatally in females (Venable, 1966). During postnatal days 7-34, testosterone treatment increases the number of multiply innervated terminals, as well as increasing the number of terminals per fiber. In Xenopus laevis, the multiply innervated type I fibers are frequent in males, but extremely rare in females. During postmetamorphic development, males are exposed to rapidly rising levels of circulating androgen; females are not (Lambdin and Kelley, 1986). If synapse elimination is androgen-sensitive in larynx, and if loss of multiple innervation results in a singly innervated high safety factor synapse, then type I fibers in the female frog may be converted to type III fibers by synapse elimination. Type I fibers would persist in males because of androgen secretion. The presence of male-typical fibers in developing female larynx has yet to be tested.

\section{Coupling between female muscle fibers}

Female laryngeal muscle fibers were dye-coupled in $8 / 20$ cases. Dye-coupling was not observed in male laryngeal muscle in any of 15 experiments. Adult female larynx contains approximately one-eighth the number of muscle fibers found in adult males (4000 versus 32,000; Sassoon and Kelley, 1986). A possible functional advantage of coupling may be to increase the number of muscle fibers contracting in response to each motor neuron action potential. This effect would, in turn, insure sufficient tension on the arytenoid discs to produce a sound. Coupled fibers are organized in sheets, each fiber (except the most medial one) positioned lateral and dorsal to its neighbor. This arrange- ment may result in a larger insertion area onto the arytenoid discs, producing more uniform and effective tension on the discs.

Dye-coupling in female laryngeal muscle may be due to gap junctions between adjacent fibers. The low-molecular-weight dye Lucifer yellow readily passes through gap junctions and has been used to indicate the presence of these membrane specializations in Xenopus laevis (Warner et al., 1984). Alternatively, muscle fibers may be dye-coupled through cytoplasmic bridges, the remnants of incomplete myocyte fusion during development. According to one view, $15 \mathrm{~nm}$ bridges between cells are formed by the incorporation of "fusiform vesicles" prior to fusion (Kalderon and Gilula, 1979). It is conceivable that complete fusion is blocked in developing female laryngeal muscle, resulting in coupled fibers. Gap junction formation has also been reported immediately preceding muscle fusion in the developing hindlimb of rats (Rash and Staehelin, 1974). In either case, dyecoupling indicates electrical continuity of fibers. The consequence for females is the synchronous contraction of many fibers.

Gap junctions have been observed in adult muscle in response to specific endocrine conditions. For example, shortly before parturition, rat uterine muscle becomes extensively coupled (Sims et al., 1982). Gap junctions can be induced in myometrial tissue by exposure to estrogens (Garfield et al., 1980). In the present study, dye-coupling was not observed in any of 8 testosteronetreated adult females examined. The presence of coupling in female laryngeal muscle is probably not due to circulating cstrogen, since this tissue does not contain estrogen receptor (U. O'Dougherty, D. A. Sassoon, and D. B. Kelley, unpublished observations). Adult female larynx does, however, contain androgen receptor (Segil et al., 1987). Thus, it is more likely that exogenously administered testosterone interferes with the maintenance or synthesis of gap junctions. Alternatively, gap junctions may be physically pulled apart in response to muscle fiber hypertrophy or to an increase in extracellular matrix, both of which have been observed in female laryngeal muscle in response to androgen treatment (Sassoon and Kelley, 1986).

The larynx is sexually monomorphic and female-like early in development. In response to continuously rising levels of androgen after metamorphosis, the male larynx gradually matures into its distinctly male-like adult form (Lambdin and Kelley, 1986; Sassoon and Kelley, 1986). In contrast, the female larynx - in the absence of androgens-retains its less developed form. Since gap junctions are a common feature of embryonic tissue, and have not been reported in adult skeletal muscle, the presence of dye-coupling between female laryngeal muscle fibers supports the hypothesis that the female larynx is indeed a less mature form of the male larynx. The finding that muscle fiber electrophysiology is also sexually dimorphic suggests that this property, too, may bc controlled by androgens during development. The physiological differences we report here may rely on motor neuron differences that appear to become nonmalleable once innervation is complete.

\section{References}

Angaut-Petit, D., and A. Mallart (1979) Dual innervation of end-plate sites and its consequences for neuromuscular transmission in muscles of adult Xenopus laevis. J. Physiol. (Lond.) 289: 203-218.

Bennett, M. R., and A. G. Pettigrew (1975) The formation of synapses in amphibian striated muscle during development. J. Physiol. (Lond.) 252: 203-239

Brown, M. C., J. K. S. Jansen, and D. Van Essen (1976) Polyneuronal 
innervation of skeletal muscle in new-born rats and its elimination during maturation. J. Physiol. (Lond.) 261: 387-422.

Garfield, R. E., M. S. Kannan, and E. E. Daniel (1980) Gap junction formation in myometrium: Control by estrogens, progesterone and prostaglandins. Am. J. Physiol. 238: C81-C89.

Grinnell, A. D., and A. A. Herrera (1980) Physiological regulation of synaptic effectiveness at frog neuromuscular junctions. J. Physiol. (Lond.) 307: 301-317.

Hannigan, P., and D. B. Kelley (1986) Androgen-induced alterations in vocalizations of female Xenopus laevis: Modifiability and constraints. J. Comp. Physiol. A 158: 517-527.

Jordan, C. L., M. S. Letinsky, and A. P. Arnold (1988) Synapse elimination occurs late in the hormone-sensitive levator ani muscle of the rat. J. Neurobiol. 19: 335-356.

Kalderon, N., and N. B. Gilula (1979) Membrane events involved in myoblast fusion. J. Cell Biol. 81: 411-425.

Kelley, D. B., S. Fenstemaker, P. Hannigan, and S. Shih (1988) Sex differences in the motor nucleus of cranial nerve ix- $\mathrm{x}$ in Xenopus laevis: A quantitative Golgi study. J. Neurobiol. 19: 413-429.

Lambdin, L. T., and D. B. Kelley (1986) Organization and activation of sexually dimorphic vocalizations; androgen levels in developing and adult Xenopus laevis. Soc. Neurosci. Abstr. 12: 1213.

Picker, M. D. (1983) Hormonal induction of the aquatic phonotactic response of Xenopus. Behaviour 84: 74-80.

Rash, J. E., and L. A. Staehelin (1974) Freeze-cleave demonstration of gap junctions between skeletal myogenic cells in vivo. Dev. Biol. 36: $455-461$.

Sassoon, D. A., and D. B. Kelley (1986) Sexually dimorphic larynx of Xenopus laevis: Development and androgen regulation. Am. J. Anat. 177: 457-472.

Sassoon, D., G. Gray, and D. Kelley (1987) Androgen regulation of muscle fiber type in the sexually dimorphic larynx of Xenopus laevis. J. Neurosci. 7: 3198-3206.

Segil, N., N. Silverman, and D. B. Kelley (1987) Androgen binding levels in a sexually dimorphic muscle. Gen. Comp. Endocrinol. 66: 95-101.

Siegel, S. (1956) Nonparametric Statistics for the Behavioral Sciences, McGraw-Hill, New York.

Simpson, H. B., M. L. Tobias, and D. B. Kelley (1986) Origin and identification of fibers in the cranial nerve IX-X complex of Xenopus laevis: Lucifer yellow backfills in vitro. J. Comp. Neurol. 244: 430444.

Sims, S. M., E. E. Daniel, and R. E. Garfield (1982) Improved electrical coupling in uterine smooth muscle is associated with increased numbers of gap junctions at paturition. J. Gen. Physiol. 80: 353-375.

Stewart, W. W. (1978) Functional connections between cells as revealed by dye-coupling with a highly fluorescent naphthalimide tracer. Cell 14: 741-759.

Tobias, M. L., and D. B. Kelley (1987) Vocalizations by a sexually dimorphic isolated larynx: Peripheral constraints on behavioral expression. J. Neurosci. 7: 3191-3197.

Venable, J. H. (1966) Morphology of the cells of normal, testosteronedeprived and testosterone-stimulated levator ani muscles. Am. J. Anat. 119: 271-302.

Warner, A. E., S. C. Guthrie, and N. B. Gilula (1984) Antibodies to gap junctional protein selectively disrupt junctional communication in the early amphibian embryo. Nature $311: 127-131$.

Weintraub, A., D. B. Kelley, and R. Bockman (1985) Prostaglandin E2 induces receptive behaviors in female Xenopus laevis. Horm. Behav. 19: 386-399.

Wetzel, D. M., and D. B. Kelley (1983) Androgen and gonadotropin effects on male mate calls in South African clawed frogs, Xenopus laevis. Horm. Behav. 17: 388-404.

Wetzel, D. M., D. Kelley, and U. Haerter (1985) A proposed neural pathway for vocalization in South African clawed frogs, Xenopus laevis. J. Comp. Physiol. 158: 749-761.

Yaeger, D. (1982) A novel mechanism for underwater sound production in Xenopus borealis. Am. Zool. 122: 887. 